Revista de Psicología de la PUCP. Vol. XIII. No 2. 1995

\title{
EL CAMBIO EN LAS CONDUCTAS ADICTIVAS
}

\author{
Luis Iván Mayor S.
}

Concita el interés de los profesionales de la salud mental el descubrir cómo se modifican los comportamientos desadaptativos, más aún existiendo gente que cambia sin tratamiento. Este artículo explica, a partir de un modelo transteórico de etapas y procesos, cómo cambia la gente. Las investigaciones, cada vez más, corroboran que en la modificación de los comportamientos existen 5 etapas: precontemplación, contemplación, preparación, acción y mantenimiento. Además los sujetos siguen procesos comunes en cada una de estas etapas.

Palabras claves: comportamientos desadaptativos, modelo transteórico, precontemplación, contemplación, preparación, acción y mantenimiento

The change in addictive behavions

Mental health professionals could be interested in finding how inappropriate behavior could be modified, specially when there are people that changes without treatment. This article explains how people change with a transteorical model of stages and processes. Psychological research shows that there are five stages in behavior modification: pre-contemplation, contemplation, preparation, action and mainrenance. Besides, subjects have common processes in every stage.

Keywords: unadaptative behavior, transtheoretical model, precontemplation, contemplation, preparation, action and mantaining

1. Magister en Psicología, Vicepresidente de la Asociación para el Desarrollo e Intervención Multidisciplinario en la Mejora de la calidad de la Vida. Miembro del Instituto Complutense de Drogadependencia. 

Schneider y Khantzian (1992) diferencian tres estadios en el proceso de abandono de una conducta adictiva:
a) Ambivalencia e indecisión
b) Resolución y acción
c) Mantenimiento y prevención de recaídas

Por último, tenemos el modelo de Prochaska y DiClemente que, aparte de su elevado valor heurístico, es el que ha recibido un soporte empírico, significativamente mayor, en relación tanto a su capacidad descriptiva como predictora del proceso de cambio. Prochaska y DiClemente (1992); Prochaska, DiClemente y Norcross (1992); Prochaska y Prochaska (1993) distinguen los siguientes estadios en el proceso de cambio de las conductas adictivas:
a) Precontemplación
b) Contemplación
c) Preparación
d) Acción
e) Mantenimiento

Este modelo es tridimensional porque proporciona una concepción global y al mismo tiempo diferenciada del cambio de las conductas adictivas, formando parte de: estadios, procesos y niveles.

Los estadios darían respuesta al cuándo suceden los cambios particulares en las actirudes, las intenciones o los comportamientos, pero con la ventaja de que, además, debido a las elevadas tasas de recaídas (Casas y Gossop, 1993; Hunt, Barnett y Branch, 1971; Marlatt y Gordon, 1985) este modelo contempla que la mayoría de individuos no progresan linealmente a través de los estadios de cambio. En este sentido, en un seguimiento de dos años en una muestra de fumadores, situados inicialmente en el estadio de contemplación Prochaska, DiClemente, Velicer, Rossi y Guidagnoli (1991) muestran cómo sólo 9 de los 180 adictos (5\%) progresaba sin recaer hasta el estadio de mantenimiento a lo largo de los dos años. En otro estudio, (Prochaska y DiClemente, 1986) la mayoría de fumadores siguen un patrón cíclico y reinciden entre tres y cuatro veces a través de los diferentes estadios hasta consolidarse en el mantenimiento. 
Cada una de las 5 etapas tiene unas características que las diferencia.

\section{Precontemplación}

\section{Características:}

* Los sujetos no quieren cambiar en un futuro próximo.

* No se dan cuenta de los problemas o los minimizan.

* Acuden a psicoterapia por la presión de otros.

* Sólo desean cambiar cuando existe una coacción o una amenaza muy fuerte.

* Quizás puedan desear cambiar pero no lo consideran seriamente.

* Usan frases como:

- Todavía no tengo ningún problema que me exija cambiar.

- Tengo fallos pero no hay nada que, en realidad, necesite cambiar.

\section{Contemplación}

\section{Características:}

* Piensan que tienen un problema pero no se han comprometido a cambiarlo.

* Pueden permanecer en esta etapa durante mucho tiempo.

* Luchan con sus evaluaciones positivas de comportamiento adictivo y pierden una gran cantidad de esfuerzo y energía para superar el problema.

* Son sujetos que quieren cambiar su comportamiento en los próximos 6 meses.

* Afirmaciones usuales:

- Tengo un problema y creo que debería trabajar en superarlo.

- He pensado que podía cambiar algo de mi.

\section{Preparación}

\section{Caracteristicas:}

* Intentan pasar a la acción el próximo mes y pasaron, sin éxito, a la acción durante el año anterior. 
* Tenían pequeños cambios, como fumar cinco cigarrillos menos, o beber sólo los fines de semana.

* Han hecho mejoras en sus problemas pero no han alcanzado el criterio de abstinencia.

\section{Acción}

\section{Caracteristicas:}

* El individuo modifica su comportamiento con acciones que implican comportamiento visibles, con un considerable compromiso de tiempo y energía.

* El cambio debe durar entre un día y 6 meses.

* Aquí debe hacer una abstinencia total de la sustancia.

* Frases:

- Estoy esforzándome mucho por cambiar.

- Hablar es fácil pero yo lo estoy haciendo.

\section{Mantenimiento}

\section{Características:}

* Se trabaja en prevenir la recaída.

* Esta aparece a partir de los 6 meses del cese de la conducta adictiva.

Prochaska y DiClemente (1984) han mencionado la existencia de un supuesto estado que pondría fin al proceso de cambio, este involucraría la total extinción de tentaciones a vencer y total seguridad de no consumir la sustancia psicoactiva. (Velicer, Prochaska, Rossi y Show, 1992).

\section{Aclarando cuestiones terapéuticas}

Es muy cotidiano que al realizar un programa de tratamiento o de autoayuda se vean pocas personas interesadas, o que muchas de las que se inscriben abandonen el programa. Esto es debido a que la mayoría de programas están dirigidos a personas que se encuentran en el estadio de Acción, pero los estudios de Abrams, Follick y Bieer (1988); Gottleib, Galarotti, McCuan y McAlister (1990) del 10 al 15\% de los fumadores están preparados para la acción; del 30 al 40\% están en la etapa de 
contemplación; y del 50 al 60\% se hallan en la etapa de precontemplación. Entonces, si los profesionales ofrecen un producto estándar indiscriminadamente, se toparán con que es ineficaz porque va dirigido sólo a un 10 o un $15 \%$ de ellos. Entonces lo que se deben hacer son programas destinados a que el sujeto progrese en las etapas de abandono hasta llegar a la etapa de acción (Prochaska y DiClemente, 1992).

Independientemente del tratamiento recibido, hay una clara relación entre la etapa de pre-tratamiento y resultados. El porcentaje de los pacientes que no fumaron durante los 6 meses siguientes al tratamiento eran un $22 \%$ precontempladores, $43 \%$ contempladores y $76 \%$ preparados para la acción o en acción.

Cuando finalizó un tratamiento para perder peso, los pacientes que antes alcanzaban la etapa de acción, antes lograban su objetivo de perder peso. La puntuación de las etapas de cambio era la más eficaz, mejor que la edad y el estatus socio-económico, la gravedad del problema y su duración, las expectativas y los objetivos, la autoeficacia y la ayuda social. Las únicas variables que caracterizan las etapas de cambio como predictoras de resultado eran los procesos de cambio que los pacientes utilizaban al principio de la terapia.

Los procesos de cambio son la segunda mejor dimensión del modelo que nos responde al cómo suceden estos cambios. Los procesos son actividades y experiencias cubiertas y abiertas con las que los individuos se comprometen cuando intentan modificar los problemas de comportamiento. Lo más interesante es que cada proceso es una amplia categoría que abarca múltiples técnicas, métodos de intervenciones, tradicionalmente asociados con dispares orientaciones teóricas. El modelo se denomina transteórico, porque identifica técnicas de cambio con diferentes teorías.

Al menos 10 componentes principales generaron puntuaciones similares deducidos al analizar los items del proceso de cambio, mediante diferentes formatos de respuesta y diferentes muestras (Norcross y Prochaska, 1986 a y b; Prochaska y DiClemente, 1983; Prochaska, Velicer, DiClemente y Fava, 1988). 
Estos procesos se evaluaron a través de autoinformes y se identificaron como veraces en las transcripciones de las sesiones de psicoterapia (O'Conell, 1989). Otro dato interesante es que personas desconocedoras del método y en proceso de cambio, utilizaron los mismos métodos que se hallan en el nudo de los sistemas de psicoterapia (DiClemente y Prochaska, 1982, 1985; Prochaska y DiClemente 1984). Entonces, existen unas actividades básicas que son las mismas y que son utilizadas, tanto por autocambiadores, pacientes en psicoterapia y profesionales de la salud mental.

Aunque existen entre 250 y 400 terapias psicológicas diferentes (Karasu, 1986) basadas en concepciones teóricas divergentes, se ha podido identificar 12 procesos diferentes de cambio, basados en los principales componentes de análisis.

Cuando se clasifican los procesos en términos de cuan frecuentemente eran utilizados para cada uno de los problemas de comportamiento. Las clasificaciones resultaron ser casi idénticas. Así, los 3 procesos con mayor puntuación en los problemas eran ayudar a relacionarse, aumentar la conciencia y la autoliberación.

Los procesos de cambio son:

Aumento de conciencia: El sujeto recolecta más información sobre sí mismo y su problema. Hace observaciones, confronta su información, hace interpretaciones, lee libros que hablen del tema.

Autoevaluación: Evalúa sus sentimientos y pensamientos respecto a su problema, clarifica valores, hace corrección de experiencias emocionales.

Autoliberación: Escoge y se compromete a actuar, creyendo en la propia capacidad de cambio. Hace ejercicios de toma de decisiones, toma resoluciones para el nuevo ańo, aplica técnicas de logoterapia y de realce de propósitos. Requiere de la creencia de que él es el elemento esencial en el cambio.

Contracondicionamiento: Se utilizan alternativas sustitutorias para problemas de conducta; relajación, desensibilización, aseveración, autoestados positivos $\mathrm{y}$, esencialmente, conductuales.

Control de estímulos: Evita o limita los estímulos que posibiliten los problemas de conducta. Restructura el propio ambiente, por ejemplo evitar 
el alcohol en casa, no ir a lugares de alto riesgo al consumo, no llevar dinero en el bolsillo.

Autogobierno: Es la vigilancia, por parte de uno mismo o por otros, en los cambios: controles de contingencia, refuerzos encubiertos, autoregistros.

Relaciones de ayuda: Ser abierto ofreciendo confianza a quien lo necesite, este es el caso de la alianza terapéutica: el soporte social del sujeto o el caso de los grupos de autoayuda.

Relieve dramático: Expresar sentimientos ante los propios problemas y dar soluciones a éstos. Estos son utilizados en el problema, en el juego de roles, etc.

Reevaluación ambiental: Como los problemas acechan en el entorno físico, entrenamiento empático, documentales.

Liberación social: Incremento de alternativas con conductas no problemáticas posibles en la sociedad; abogar por el derecho a reprimirse; autorización intervenciones en la línea de conducta.

Se pueden integrar los estados o etapas de cambio a los procesos. Así tenemos que en el estadio de precontemplación los adictos utilizaban significativamente más procesos de cambio que aquellos sujetos que se hallan en cualquier otro estadio. Los precontempladores, entonces:

* Procesan menos información referente a su problema.

* Utilizan menos tiempo y energía reevaluándose a si mismos.

* Experimentan menos reacciones emocionales frente a los aspectos negativos de su adicción.

* Son menos abiertos y comunicativos respecto a su problema.

Los sujetos en la etapa de contemplación están más abiertos a las técnicas:

* Aumentar la conciencia.

* Hacer confrontaciones e interpretaciones.

* Utilizar biblioterapia y demás técnicas educaciones.

Los contempladores tienen más procesos de conciencia de relieve dramático y reevaluación ambiental. Hay más autoreevaluación por estar el 136 
paciente entre la contemplación y la preparación donde se enfatiza más la autoliberación, enlazándose con el estadio de acción.

En los estadios de acción y mantenimiento tienen los procesos de relevos de ayuda, manejo de contingencias, control de estímulos y contracondicionamiento.

Prochaska, Velicer, DiClemente, Guadagnoli y Rossi (1991) han identificado que tres de los cinco procesos cognitivos experienciales (aumento de la concienciación, relieve dramático y reevaluación ambiental) presentan un patrón curvilíneo común. Estos 3 procesos de cambio son muy poco utilizados en el estado de preparación; para aumentar cuando se progresa de la contemplación a la acción, llegado a su punto máximo. Luego disminuye en su frecuencia de utilización durante los estadios de acción y mantenimiento hasta niveles casi precontemplativos.

A medida que los sujetos progresan hacia el estadio de mantenimiento la utilización del proceso de autoreevaluación disminuye gradualmente hasta niveles de precontemplación.

El proceso de liberación social es el único que no sigue un perfil curvilíneo, su punto más elevado está en el estadio de precontemplación y disminuye progresivamente entre este estadio y el mantenimiento.

El proceso de manejo de contingencias alcanza su punto más elevado en la fase inicial del estadio de acción, para disminuir gradualmente durante el mantenimiento.

Ninguno de los procesos más conductuales disminuye hasta niveles precontemplativos.

La tercera dimensión del modelo transteórico de Prochaska y DiClemente nos explica qué cambios se necesitan para abandonar una conducta adictiva.

En 5 niveles se organizan jerárquicamente las diferentes evaluaciones $e$ intervenciones terapéuticas:

1) Síntoma/situacional

2) Cogniciones desadaptadas 
3) Conflictos interpersonales actuales

4) Conflictos sistémicos/familiares

5) Conflictos intrapersonales

Como sugieren Prochaska y DiClemente (1986); DiClemente, McConaughy, Norcross y Prochaska (1986) se prefiere intervenir a nivel síntoma/situacional por cuatro motivos principales:

a) El cambio tiende a suceder con más facilidad en este nivel más manifiesto y observable.

b) Este nivel generalmente representa el motivo principal del adicto que acude a tratamiento.

c) Puesto que el nivel es el más consciente y contemporáneo, el grado de interferencia necesaria para una evaluación e intervención clínica es menor.

d) Dado que estos niveles no son independientes, el cambio en uno de ellos probablemente provoca cambios en otros.

El equipo de Prochaska (1991), después de investigar con poblaciones de fumadores, bebedores y con trastornos de peso, ha descubierto unos patrones comunes de cómo la gente modifica su comportamiento. Estos experimentos, entonces, justifican una concepción transteórica, un patrón crítico de movimiento a través de las etapas de un grupo común de procesos de cambio. Una sistemática integración de las etapas y de los procesos de cambio.

La implicación más importante y directa de este modelo es la necesidad de evaluar la etapa en la que se encuentra el paciente en su disposición al cambio y ajustar las intervenciones a su medida.

Otra conclusión que se puede desprender es que un autocambiador eficiente hace más cosas correctamente (procesos) en el momento preciso (etapas).

El error en el que caen muchos sistemas de psicoterapia es hacer más conscientes a sus pacientes acerca de su problema cuando se encuentran en la etapa de acción, sólo Insight no aporta un cambio de conducta. Por otra parte se le critica al conductismo radical, el no aplicar el requerido 138 
análisis y toma de decisión, proporcionado en las etapas de contemplación y preparación. Tratar de modificar la conducta sin esa acción abierta, sin introspección es probable que conduzca a cambios sólo temporales.

Parecen utilizarse procesos similares para modificar diversos problemas y se utilizan, entre y sin sesiones de psicoterapia, por eso a los sujetos que se les apliquen procesos oportunos, tienen garantizado el éxito en la terapia, siempre y cuando se tenga en cuenta que los sujetos reinciden varias veces a través de las etapas antes de conseguir un mantenimiento de larga duración.

\section{Referencias}

Abrams, D.D. Follick, M.J., y Bieer L. (1988). Individual versus group self-help smoking cessation at the workplace: Initial impact and 12 month outcomes. En T. Glynn (Chair), National Cancer InstituteFunded self-help smoking cessation trials: Interim results and emerging patterns. Simposio de la reunión anual de la Association for the Advancement of Behavior therapy. New York.

Brownell, K.D.; Marlatt, G.A.; Lichtenstein, E. y Wilson, G.T. (1986). Understanding and preventing relapse. American Psychologist, 41, 765782.

Casas, M. y Gossop, M. (Eds.), (1993). Tratamientos psicológicos en drogodependencias: recaida y prevención de recaídas. Sitges: Ediciones en Neurociencias.

Cohen, S.; Lichtenstein, E.; Prochaska, J.O.; Rossi, J.S.; Gritz, E.R.; Carr, C.R.; Orleans, C.T.; Schoenbach, V.J.; Biener, L.; Abrams, D.; DiClemente, C.C.; Curry, S.; Marlart, G.A.; Cummings, K.M.; Emot, S.L.; Giovino, G. y Ossipklein, D. (1989). Debunking myths about self-quitting: Evidence from 10 prospective studies of persons quitting smoking by themselve. American psychologist 44, 1355-1365.

DiClemente, C.C. y Prochaska, J.O. (1982). Self-change and therapy change of smoking behavior: A comparison of processes of change of cessation and maintenance. Addictive Behaviors, 7, 133-142.

DiClemente, C.C. y Prochaska, J.O. (1985). Processes and stages of selfchange: Coping and competence in smoking vehavior change. En $S$. 
Shiffman y T.A. Wills (Eds.). Coping and substance use (pp. 319-343). Londres: Academic Press.

DiClemente, C.C.; McConnaughy, E.A.; Norcross, J.C. y Prochaska, J.O. (1986). Integrative dimensions for psychotherapy. International Journal of Electric Psychotherapy, 5, 256-274.

Gottlieb, N.H.; Galavotti, C.; McCuan, R.A. y McAlister, A.L. (1990). Specification of a social-cognitive model predicting smoking cessation in a Mexican-American populations: A prospective study. Cognitive Therapy and Research, 14, 529-542.

Hunt, W.A.; Barnett, L.W. y Branch, L.G. (1971). Relapse rates in addiction programs. Journal of Clinical Psychology, 27, 455-456.

Karasu T.B. (1986). The specificity versus nonspecificity dilemma: Toward identifying therapeutic change agents. American Journal of Psychiatry, 143, 687-695.

Lambert, M.J.; Shapiro, D.A. y Bergin, A.E. (1986). The effectiveness of psychotherapy. In S.L. Garfield y A.E. Bergin (Eds.). Handbook of psychotherapy and behavior change (3ra. ed.). Nueva York: Wiley.

Marlatt, G.A. y Gordon, J.R. (1985). Relapse prevention. Maintenance strategies in the treatment of addictive vehaviors. Nueva York: Guildford Press.

Marlatt, G.A.; Baer, J.S.; Donovan D.M. y Kivlahan, D.R. (1988). Addictive behaviors: Etiology and treatment. Annual Review of Psychology, 39, 223-252.

Norcross, J.C. y Prochaska, J.O. (1986 a). Psychotherapists heal theyself - I. The psychological distress and self-change of psychologists, counselors, and laypersons. Psychotherapy: Theory, Research, and Practice, 23, 102-114.

Norcross, J.C. y Prochaska, J.O. (1986 b). Psychotherapists heal theyself - II. The self-initiated and therapy facilitated change of psychological distress. Psychotherapy: Theory, Research, and practice, 23, 345-356.

O'Connell, D. (1989). An observational coding scheme for therapists' processes for change. Unpublished doctoral dissertation. University of Rhode Island: Kingston.

Orford, J. (1985). Excessive Appetites: A Psychological View of Addictions. Nueva York: John Wiley and Sons.

Prochaska, J.O. (1991). Prescribing to the stages and levels of change. Psychotherapy, 28, 463-468. 
Prochaska, J.O. y DiClemente, C.C. (1983). Stages and processes of selfchange of smoking: Toward and integrative model of change. Journal of Consulting and Clinical Psychology, 51, 390-395.

Prochaska, J.O. y DiClemente, C.C. (1984). The transtheoritical approach: Crossing the traditional boundaries of therapy. Homewood. Illinois: Dorsey Press.

Prochaska, J.O. y DiClemente, C.C. (1986). Toward a comprehensive model of change. En W.R. Miller y N. Heather (Eds.). Treating addictive behaviors: Processes of change, Nueva York: Plenumm Press. Prochaska, J.O. y DiClemente, C.C. (1992). Stages of change in the modification of problem behaviors. En M. Hersen; R.M. Eisler y P.M. Miller (Eds.). Progress in behavior modification. Newbury Park. California: Sage.

Prochaska, J.O.; DiClemente, C.C. y Norcross, J.C. (1992). In search of how people change. Applications to addictive behaviors. American Psychologist, 47, 1102-1114.

Prochaska, J.O.; DiClemente, C.C.; Velicer, W.F.; Rossi, J.S. y Guadagnoli, E. (1991). Patterns of change II: Longitudinal analysis of self-change processes across stages of smoking cessation. Manuscrito no publicado (citado por Velicer, Prochaska, Rossi y Snow, 1992).

Prochaska, J.O.; Velicer, W.F.; DiClemente, C.C. y Fava, J. (1988). Mesasuring processes of change: Applications to the cessation of smoking. Journal of Consulting and Clinical Psychology, 56, 520-528.

Prochaska, J.O.; Velicer, W.F.; DiClemente, C.C.; Guadagnoli, E. y Rossi, J.S. (1991). Patterns of change: Synamic typology applied to the smoking cessation. Multivariate Behavioral Research. 26, 83-107.

Prochaska, J.O. y Prochaska, J.M. (1993). Modelo trans-teórico de cambio de conductas adictivas. En Casas y M. Gossop (Eds.). Recaida y prevención de recaídas. Tratamientos psicológicos en drogodependencias. Barcelona: Ediciones de Neurociencias. Citrán. FISP.

Raw, M. (1986). Smoking cessation strategies. En W.R. Miller y N. Heather (Eds.). Treating addictive behaviors: Processes of change. Nueva York: Plenum Press.

Roizen, R. Cahaland, D., y Shanks, R. (1978). Spontaneous remission among untreated problem drinkers. In Randell (Ed.), Longitudinal research on drug use empirical findings and methodological Issues, Washington, D.C.: Hemisphere. 
Rosen, T.J. y Shipley, R.H. (1983). A stage analysis of self-initiated smoking reduction. Addictive Behaviors, 8, 263-272.

Schneider, R.J. y Khantzian, E. (1992). Psychotherapy and patient needs in the treatment of alcohol and cocaine abuse. En M. Galanter (Ed.). Recent developments in alcoholism. Nueva York: Plenum Press.

Shaffer, H.J. (1992). The psychology of stage change: The transition from addiction to recovery. En J.H. Lowinson; P. Ruiz; R.B.; Millman y J.G. Langrod (Eds.). Substance abuse: A comprehensive textbook (2da. ed.). Baltimore: Williams and Wilkins.

Smith, M.L.; Glass, G.V. y Miller, T.I. (1980). The benefits of psychotherapy. Baltimore: Johns Hopkins University.

Tushfeld, B. (1981). Spontaneous remission in alcoholics: Empirical observations and theoritical implications. Journal for Studies on Alcohol, 423, 626-641.

Velicer, W.F.; Prochaska, J.O.; Rossi, J.S. y Show, M.G. (1992). Assessing outcome in smoking cessation studies. Psychological Bulletin, 111, 2341. 